\title{
De Bartolomé de Las Casas al TLC, pasando por Multatuli
}

\author{
Victor Valembois \\ Universidad de Costa Rica
}

\section{Resumen}

Se propone una lectura en clave política, y desde las condiciones del presente, de la breve novela Max Havelaar, del holandés Eduard Douwes Dekker (Multatuli). Plantea un parangón entre las reivindicaciones y tesis políticas que el relato despliega, con los escritos de Bartolomé de Las Casas a propósito de la colonización: además, establece vínculos significativos con la situación política centroamericana contemporánea.

\section{Abstract}

An interpretation is proposed here in political terms-from the perspective of the present —of the short novel Mar Havelaar by the Dutch author Eduard Douwes Dekker (Multatuli). The demands and political theses of the novel are compared with the writings of Bartolomé de Las Casas on colonization; in addition, significant ties are developed with the contemporary political situation in Central America.

* En primera versión, este texto constituyó una ponencia para el Encuentro de la Regional Humanista Latinoamericana. 27-29 de mayo 2004 en San José, Costa Rica. 
No solicito nada para mí mismo sino para la causa que defiendo, la causa de la justicia y del humanismo que es la misma que la causa de un ilustrado auto-interés. Multatuli $^{1}$

Para Ester Previtera, otra trashumante, en pro de nuestras luchas por más humanidad en todas partes.

\section{Hace ciento cincuenta años, desde Bruselas, con amor}

No viene el mensaje desde Rusia esta vez, como en esa película taquillera. "Sufrí una barbaridad" se nos queja su autor, y lo hace desde Bruselas, casi con lágrimas. ¡Cómo no llorar de rabia! Se trata de un joven funcionario en el sistema colonial holandés que, por honesto, denuncia la corrupción tanto del lado de reyezuelos indonesios, como de sus propios compatriotas en los Países Bajos. Por no tener pelos en la lengua, lo despiden y allí lo tienen, en una especie de autoexilio, sacando punta al lápiz desde la capital de Bélgica. Es una historia vieja, pero como en todas partes cuecen habas... e injusticias, allí va.

Por cierto, respecto de la decisión de alejarse de la patria, no pareciera que se impusieron razonamientos políticos ni ideológicos, en Eduard Douwes Dekker (1820-1874), el "espía que vino del frío" del corazón. Bélgica llevaba entonces apenas unas décadas de vida soberana al haberse separado de Holanda en 1830. Históricamente esos dos países tenían mucho en común como "Países Bajos", pero la despiadada ocupación española, represiva en lo militar, lo cívico y lo religioso $^{2}$, había llevado a que tuvieran cada uno por su lado su

1. En mis citas, me basaré en la edición inglesa, Max Havelaar or the coffee auctions of the Dutch Trading Company (Nueva York: Penguin Classics, 1967) 317. En neerlandés: Multatuli, Max Havelaar of de koffieveilingen der Nederlandse handelsmaatschappij (Amsterdam: L. J. Veen-Ede, 1979). Ignoro la existencia de una traducción española.

2. En varios momentos se alude a ello en la novela: "El Duque de Alba era un monstruo", señala Droogstoppel, p. 20; otra alusión, p. 45, etc. 
evolución. El divorcio de los dos, artificialmente unidos por los grandes a consecuencia de la derrota de Napoleón, en 1815, no hizo sino confirmar la separación iniciada en el siglo $\mathrm{xvI}^{3}$.

Pero volvamos al relato que escribió ese inconforme; lo elaboró no en película sino en vivos colores de denuncia. Lo más probable es que, simplemente como en tantos casos documentados, la escogencia de Bruselas para escribir ese llamado, a veces violento y hasta vitriólico, por elemental justicia, fue por lo cerca de su país, al mismo tiempo que por lo económico ${ }^{4}$ : salía más barato alojarse en una buhardilla en la capital de Bélgica que, en este caso, en Holanda. Como en casos memorables de Victor Hugo hasta Prigogine, pasando por Marx y Van $\mathrm{Gogh}^{5}$, Bruselas se constituía otra vez en paño de lágrimas en el ámbito europeo.

La misma novela varias veces refiere a Bélgica ${ }^{6}$ y por cierto otros diversos factores biográficos relacionan también al novelista que nos ocupa con Bélgica y particularmente con su capital ${ }^{7}$. En su propia introducción a la edición de 1875 , el autor evoca de manera tan clara como conmovedora las circunstancias en que le tocó trabajar:

3. Sobre la relación Bélgica-Holanda y, más concretamente, su incidencia idiomática de dos variantes regionales en una misma lengua. ver "El neerlandés, la lengua de más de veinte millones de europeos", primer capítulo de un libro mío en preparación.

4. Confirmación de ello da Lode Craeybeckx. en Cent ans de démocratie bourgeoise: "en Bélgica la vida resulta más barata que en otra parte, sobre todo en lo que se refiere a alojamiento" (79). Esa es también la razón por la cual gran cantidad de médicos, y profesionales costarricenses en general, estudiaron allá. Ver: "Una generación única de profesionales ticos. formados en Bélgica", Herencia 7-8, n 1-2, 1995-96. pp. 15-26.

5. En su "Introducción por el traductor", Roy Edwards apunta varias similitudes entre Van Gogh y Multatuli. Curiosamente pasa por alto otra, quizá la más importante: los dos empezaron su involuntaria carrera artística en Bélgica. Tengo un artículo en preparación al respecto.

6. Son todas alusiones secundarias, como una a Indépendance (60), un periódico francóf ono en el que trabajó por un tiempo el mismo Multatuli, en Bruselas; de repente se habla de Waterloo (111).

7. El lector interesado se sorprenderá con la cantidad de "puentes" biográficos entre Multatuli y Bélgica. Por avatares del exilio, tuvo que ver con Amberes, Spa, Lieja y sobre todo Bruselas. Roy Edwards, con insistencia, retoma dos veces el punto, p. 7: "empezó a escribir en Bruselas" y después, "en Bruselas volvió a escribir", aludiendo a que allí se puso a adaptar para su novela elementos anteriores dispersos, como una obra de teatro, un diario ficticio y algunos poemas. También consta que su familia era de flamencos antiguos que emigraron al norte con motivo de la represión colonialreligiosa española del siglo XVI en los históricos "Países Ba jos". 
... escribí mi Max Havelaar en Bruselas en el invierno de 1859, en parte en un pequeño cuarto sin calefacción, en parte en una miserable y sucia taberna en medio de gente tomando cerveza. Eran personas de buen ánimo pero no precisamente inclinadas hacia las artes. Yo estaba empecinado por llevar a cabo algo, terminar algo, lograr algún producto. La esperanza me dio ánimo... (p. 321, lo destacado es por parte del autor).

A los ciento cincuenta años, esa tea de esperanza por justicia y humanismo, prendida en Bruselas (punto 1) para nada se ha apagado; al contrario, encuentra aplicación en luchas contemporáneas, incluyendo el mismo Tratado de Libre Comercio (punto 4). Ahora bien, la batalla librada por vía de lo escrito, de parte de su autor, encuentra sorprendentes puntos de afinidad con la de Bartolomé de Las Casas (punto 2); al mismo tiempo, también se distingue de él en una cantidad de aspectos que lo vuelven aun más vigente (punto 3 ).

Mi propósito en este primer aporte sobre la misma novela es entonces ilustrar esos aspectos, específicamente para centroamericanos. Desde luego, pocos resultan familiarizados con el pensamiento de este autor, más conocido por su seudónimo Multatuli (latinismo por "he sufrido mucho"). Aclararé el porqué de ese sufrimiento y a partir de ahora me voy a referir al autor con ese alias que lo hizo famoso... y justiciero. En un segundo aporte, compararé la novela con otra, de corte antiimperialista, producida precisamente en Centroamérica: Mamita Yunai; se verá además que aquello que en francés a veces, con ironía llaman como "le reste est littérature" (lo demás es literatura), a la postre no resulta tan inútil.

\section{Un Bartolomé de nuevo cuño}

Para los de mi idioma materno, el neerlandés (en sus variantes de holandés y flamenco), Max Havelaar, es una obra artística emblemática, con resonancia nacional. Como el Quijote en el mundo 
hispano. Por cierto, sobre todo para el lector europeo, y más, para el hispanohablante, salta a la vista el parangón con esta última novela. Por ejemplo, en su introducción a la edición que manejo, el Profesor Meijer se refiere acertadamente al "sentimiento romántico-quijotesco" (5) que animó al autor. Igual, dentro de la creación, el narrador se refiere cuatro veces al héroe español, cosa en sí significativa para un nórdico, sobre todo hace tanto tiempo, cuando no era tan evidente asomarse al balcón literario del sur.

La primera mención consiste en una breve referencia al episodio de "La boda de Camacho"(36), de pasada en la obra que comentamos. En la segunda aprendemos que Havelaar era "caballeroso y valiente, pero como el otro don Quijote gastó su energía contra molinos de viento" (89). Esta comparación tiene un gran peso en sí y a todas luces revela mucho de autorretrato, de afinidad y hasta identidad entre el hablante literario y el escritor. La tercera señala que (sus enemigos, del holandés) le llaman Quijote, pero al mismo tiempo lo que les interesa (a ellos) es que sus propios molinos giren a todo dar (129), es decir, en vez de "enderezar entuertos", se aprovechan de ellos: ampliaremos sobre este tema luego. La última referencia consiste en que el narrador refiere a Indonesia como "Insulinda" (246): claro invento paralelo a aquella Isla Barataria cervantina. Solo que asistimos a una inversión: en el Quijote, se trataba de un lugar imaginario bueno, mientras la novela Max Havelaar se ubica en una isla real y pérfidamente mala, no por ella, sino por sus administradores.

Pero, ya que mi propósito aquí es vivenciar esta obra para centroamericanos, me urge probar que ese Max Havelaar también se parece a otra figura española, esta vez no de invención artística sino histórica: antes que Cervantes creara su personaje, ¿no fue Bartolomé de Las Casas (1484-1566) un verdadero Quijote en Centroamérica? En ninguna parte de la novela del holandés, ni de los comentarios en forma de introducción a mi edición, se alude a este preclaro español, pero desde este lado del Atlántico, se me evidencia el parangón en cinco rubros principales: 
Concepto humanista del otro

Los dos protohombres europeos parten de un concepto humanista y universal, para el cual todos debemos comprometer todavía nuestro mejor empeño. Lo mismo que el "Apóstol de los indios" (en este caso, de los mesoamericanos), en vez de andar en disquisiciones teológicas sobre si ellos tienen alma o no, este nuevo "Apóstol de Indonesia" se pone a la tarea de reconocerlos como seres humanos. Obras son amores, y si toda la vida se le va en una noble causa, mejor. Multatuli profesa de hecho la-original idea lascasiana. De allí el tan vigente propósito del novelista: "me ocuparé de hombres y mujeres, de seres creados que viven y se mueven y piensan igual que nosotros hacemos" (245). Partimos de nuestra radical condición de seres vivos, que amamos y sufrimos: punto de vista simplemente humanista, anhelo también del presente congreso.

El autor no postulaba que todos pensáramos igual, al contrario. afirma y fomenta que los otros se expresaran de manera diversa. De vivir ahora sería ferviente partidario de la polifonía cultural. Lo anterior iba y va pareado con una variopinta gama de colorcitos de piel. Multatuli conmina también a sus propios compatriotas para que apliquen de otro modo sus cualidades "blancas" (246). Así, tanto el autor. como Las Casas, resultan mucho más que, respectivamente. un funcionario colonial holandés o un misionero español: son antropólogos. los dos, al rescate de una cultura oprimida. En ésta va, desde luego. su condición económica, pero también su expresión humana. lo artístico incluido: ver la conocida historia de Saijah y su amor imposible por Adinda (la conmovedora historia se narra en el capítulo 17).

Lo anterior representa una joya de la literatura universal, digna de ser comparada con Tristán e Isolda o Romeo y Julieta. En su arrebato emocional, Multatuli parece justificar su inventiva al señalar que "hay muchas Adindas y hay muchos Saïjahs (...) [y] solo sugerí lo que les estaba pasando en el corazón" (278). ¡Poderosa sugerencia, en fondo y en forma! Como equivalente, se nos viene inmediatamente 
a la mente el gran Bartolomé convirtiendo a los indios de Guatemala al compás de la música... Él logró sensibilizar por las artes a esos habitantes de Verapaz. Ahora bien, ¿seremos nosotros inferiores en entendimiento sensible como para no dejarnos impactar por la poesía dramática que narra Saïjah?

\section{La rebeldía contra la esclavitud}

Cuán grande fue la similitud Las Casas-Multatuli, se comprueba en su paralela lucha contra un sistema literalmente de esclavitud, disfrazada de encomienda, en el primer caso, y de "plantación" en el segundo. La alarma se enciende de la misma manera en ambos casos, al comprobar, uno en Centroamérica, otro en Indonesia, lo que ocurre con la población local, desde lo demográfico. Multatuli constata: "el declive de la población de Parang-Kujang se debe únicamente a la manera ultrajante en que se explota a la población" (130). En términos médico-religiosos que no le eran ajenos al gran Bartolomé, denuncia "ese cáncer de la labor sin paga" (205), una encomienda de nuevo cuño.

Y he aquí de nuevo, por parte de esos europeos de vanguardia, la capacidad de destacar por encima de su entorno, al estar su gente aferrada a un punto de mira únicamente occidental. Señala Multatuli: “...pocos europeos piensan que vale la pena agacharse y observar los sentimientos de esas máquinas para producir café y azúcar que llamamos 'indígenas"' (279) y recalca: "me da lo mismo ser considerado un artista incompetente, siempre y cuando se admita que el trato dado a los nativos es ESCANDALOSo" (280: las mayúsculas son del autor). Por ello, sus escritos, su esfuerzo personal, de los dos, no resultaron en vano: la abolición del sistema de encomienda y las "Nuevas Leyes de Indias", de 1542, son el resultado conseguido por Las Casas; mejoras para los nativos también se presentaron gracias al tesón del holandés. 
Hombres con temple, como pocos

Por muy pacíficos que fueron los dos héroes, al no recurrir a la vía opresiva y si es del caso, militarista, ambos se caracterizaron por un carácter fuerte, intransigente en defensa de sus principios. Es históricamente conocido que la personalidad, tanto de Las Casas como de Multatuli, era de tenacidad, de "armas tomar". Pero la vehemencia con que defendieron sus criterios y sus actos jamás ha de confundirse con violencia. De allí, su también conocida testarudez. Eran hombres de una sola pieza: el español abandona la comodidad de su hacienda para hacer votos religiosos, como dominico; durante su larga vida cumplió con creces con el juramento de "proteger a la población nativa contra la opresión, el maltrato y la extorsión" (104) que se señala en Max Havelaar. Se lo tomaron en serio. En paralelo al Defensor de los Indios, Multatuli gritó: "yo cumplí con mi deber, mi deber completo y nada más que mi deber" $(301,302,316)$. En la biografía de los dos ha habido tremendos momentos de soledad, pero lo más importante es que su sufrimiento tenía orientación altruista.

\section{Crítica al propio gobiemo}

Bartolomé de Las Casas fue el primero en criticar la opresión de los nativos americanos por sus conquistadores europeos. Ya en 1515 le llamó la atención, directamente, al rey Fernando V (de Aragón, el esposo de Isabel, la Reina Católica) y un año más tarde retornó a Hispaniola como protector oficial de los indios. El dominico se había propuesto viajar a Flandes a hablar con el nuevo rey, el flamenco Carlos V, pero por suerte logró ver a su influyente asesor en Madrid, guardando de él un excelente recuerdo ${ }^{8}$.

8. Cito por el estudio de Juan Durán, Bartolomé de Las Casas ante la conquista de América. Las voces del historiador (Heredia: EUNA, 1992) 34. 
De un modo paralelo, Multatuli no le tiene miedo a acudir a las altas esferas: su texto va dedicado a su Rey, al cual alude dentro de la novela: "Vea, Majestad, es lo que está pasando en su imperio, ¡en su adorado Imperio de Insulinda!" (246). Terrible resulta la acusación del funcionario estatal contra sus propios superiores: el gobierno sabe que campea la corrupción, porque él mismo la provoca, al dejar a las autoridades locales parte de las ganancias, a costa del hambre de los indígenas; pese a todo, denuncia el autor, uno estaría por aplaudir el humanismo que desborda de los informes oficiales (74-75). Y sin pelo en la lengua afirma: "los informes oficiales de los funcionarios al gobierno (...) en gran parte son falsos" (destacado del autor, 213). De manera insistente denuncia el "falso optimismo" o el "optimismo artificial" (213 y 228). Está al borde de la desesperación cuando postula: "es mucho más fácil para ciertas autoridades reducir a un oficial europeo a ser mendigo que de castigar a un jefe nativo" (233).

\section{Otros puentes comparativos}

La comparación entre las dos figuras ejemplares puede prolongarse: ambos eran hijos de mercantes, gente abierta a otros mundos. Los dos tenían un "destino manifiesto" como funcionarios imperiales, pero de repente, al estilo de San Pablo, senda "conversión" los hace cambiar de rumbo y defender a los que la "lógica" del sistema incitaba a explotar; habían recibido una excelente formación clásica (con latín y griego), que los capacitaba a ser vástagos ejemplares, además de excelentes guardianes del Estado que los mandó. Ninguno tenía vocación ni preparación literaria, y sin embargo, de repente se pusieron a escribir, frenéticamente; con su respectiva pluma bien afilada, ambos emprendieron una cruzada. Con rabia, Las Casas escribió su Brevísima relación de la destrucción de Indias (1552), manifiesto clave, de inmediato traducido en varios idiomas9; paralelamente, en

9. En su estudioDurán Luzio apunta que "sólo en el siglo XVI la Brevísima relación tiene (...) traducciones al holandés: [en] 1578, 1579 y 1596". (Nota 2 de su capítulo IV). 
Hombres con temple, como pocos

Por muy pacíficos que fueron los dos héroes, al no recurrir a la vía opresiva y si es del caso, militarista, ambos se caracterizaron por un carácter fuerte, intransigente en defensa de sus principios. Es históricamente conocido que la personalidad, tanto de Las Casas como de Multatuli, era de tenacidad, de "armas tomar". Pero la vehemencia con que defendieron sus criterios y sus actos jamás ha de confundirse con violencia. De allí, su también conocida testarudez. Eran hombres de una sola pieza: el español abandona la comodidad de su hacienda para hacer votos religiosos, como dominico; durante su larga vida cumplió con creces con el juramento de "proteger a la población nativa contra la opresión, el maltrato y la extorsión" (104) que se señala en Max Havelaar. Se lo tomaron en serio. En paralelo al Defensor de los Indios, Multatuli gritó: "yo cumplí con mi deber, mi deber completo y nada más que mi deber" $(301,302,316)$. En la biografía de los dos ha habido tremendos momentos de soledad, pero lo más importante es que su sufrimiento tenía orientación altruista.

\section{Crítica al propio gobiemo}

Bartolomé de Las Casas fue el primero en criticar la opresión de los nativos americanos por sus conquistadores europeos. Ya en 1515 le llamó la atención, directamente, al rey Fernando V (de Aragón, el esposo de Isabel, la Reina Católica) y un año más tarde retornó a Hispaniola como protector oficial de los indios. El dominico se había propuesto viajar a Flandes a hablar con el nuevo rey, el flamenco Carlos V, pero por suerte logró ver a su influyente asesor en Madrid, guardando de él un excelente recuerdo ${ }^{8}$.

8. Cito por el estudio de Juan Durán, Bartolomé de Las Casas ante la conquista de América. Las roces del historiador (Heredia: EUNA, 1992) 34. 
De un modo paralelo, Multatuli no le tiene miedo a acudir a las altas esferas: su texto va dedicado a su Rey, al cual alude dentro de la novela: "Vea, Majestad, es lo que está pasando en $s u$ imperio, ¡en su adorado Imperio de Insulinda!"'(246). Terrible resulta la acusación del funcionario estatal contra sus propios superiores: el gobierno sabe que campea la corrupción, porque él mismo la provoca, al dejar a las autoridades locales parte de las ganancias, a costa del hambre de los indígenas; pese a todo, denuncia el autor, uno estaría por aplaudir el humanismo que desborda de los informes oficiales (74-75). Y sin pelo en la lengua afirma: "los informes oficiales de los funcionarios al gobierno (...) en gran parte son falsos" (destacado del autor, 213). De manera insistente denuncia el "falso optimismo" o el "optimismo artificial" (213 y 228). Está al borde de la desesperación cuando postula: "es mucho más fácil para ciertas autoridades reducir a un oficial europeo a ser mendigo que de castigar a un jefe nativo" (233).

\section{Otros puentes comparativos}

La comparación entre las dos figuras ejemplares puede prolongarse: ambos eran hijos de mercantes, gente abierta a otros mundos. Los dos tenían un "destino manifiesto" como funcionarios imperiales, pero de repente, al estilo de San Pablo, senda "conversión" los hace cambiar de rumbo y defender a los que la "lógica" del sistema incitaba a explotar; habían recibido una excelente formación clásica (con latín y griego), que los capacitaba a ser vástagos ejemplares, además.de excelentes guardianes del Estado que los mandó. Ninguno tenía vocación ni preparación literaria, y sin embargo, de repente se pusieron a escribir, frenéticamente; con su respectiva pluma bien afilada, ambos emprendieron una cruzada. Con rabia, Las Casas escribió su Brevísima relación de la destrucción de Indias (1552), manifiesto clave, de inmediato traducido en varios idiomas ${ }^{9}$; paralelamente, en

9. En su estudio Durán Luzio apunta que "sólo en el siglo XVI la Brevísima relación tiene (...) traducciones al holandés: [en] 1578, 1579 y 1596". (Nota 2 de su capítulo IV). 
Max Havelaar, Multatuli sacó de sus entrañas todo lo que le pesaba e, igual, su trabajo rápidamente fue conocido en el ámbito internacional.

\section{Multatuli, heredero de Bartolomé, tan contemporáneo de nosotros}

Más allá de tiempos y espacios, hemos podido observar cuán afín resultan las luchas humanísticas de Bartolomé de Las Casas y de Multatuli. Es probable que no se pueda hablar de influencia, sino que está de por medio la fuente común de la civilización europea. Hasta los grandes personajes, en su pensar y actuar suelen ser hijos de su tiempo: inevitable circunstancia orteguiana. Ésta, desde luego incluye también el pasado y desde este punto de vista, el antecedente español debe ser que hasta inconscientemente haya servido de soporte para el holandés.

Sin embargo, en el caso de Multatuli se pueden visualizar por lo menos cuatro grandes puntos de diferencia, en superación de Las Casas, lo impresionante del caso y lo que, filólogos o no, a todos nos puede interesar todavía del pensamiento de ambos, en nuestras luchas contemporáneas. A partir de Max Havelaar, sigo entonces ilustrando específicamente al holandés. A pesar de siglo y medio ya que nos separa de él, se comprobará cuán grande resulta su afinidad con el pensamiento crítico actual respecto de la utopía humanista.

\section{Contra las medias tintas, postura individual}

A lo largo de la novela, Multatuli reacciona con energía contra la falsedad de las medias tintas. En una apasionada protesta contra la mediocridad exclama: "La falta de compromiso no lleva a nada. Medio bueno no es bueno; verdades a medias son mentiras. Por un salario completo (...) después de un juramento claro y completo, uno queda comprometido a hacer completamente su tarea" (229 y así, en varias partes: de nuevo, los destacados son del autor). Esta postura, opuesta 
totalmente a la doble moral, al divorcio entre el decir y el hacer, constituye uno de los pilares de su obra y... perdonen la franqueza, su aplicación vendría bien en cierto país centroamericano de cuyo nombre no quiero acordarme. En comparación con la época de Las Casas, no es que él no denuncia a hipócritas, pero la forma generalizada e irónica en que lo hace Multatuli le da un vuelo superior, en ese aspecto.

En la obra artística Max Havelaar, el que caracteriza esta actitud, tan cómoda como ambigua, es un personaje de nombre Droogstoppel. Constituye el prototipo de la gente "bien pensante". Recalca siempre lo suyo como normal, es decir moral, con el sentido común y lo correcto... de su lado. Constantemente refiere a algo de la Biblia y a cada rato siente la necesidad de subrayar, como otro leitmotiv, que él obedece a principios $(28,54,55,252,289,291, \ldots)$, aunque su conducta, visualizada a lo largo de los mismos capítulos, dista mucho de esos predicados. En fin, es un oportunismo rampante. De él es el tipo de raciocinio deductivo, según el cual, "los pobres son pobres por su culpa (...) el Señor no abandonaría a nadie que lo venere con tanta fe" (28). Especialmente sarcásticas resultan sus opiniones sobre el arte y los artistas. En realidad, la dicotomía DroogstoppelHavelaar es el soporte crucial de toda la novela: los dos aparecen como narradores, el primero incluso en los cuatro capítulos de entrada, pero el lector inteligente rápidamente sabrá que se trata de un recurso literario, cargado de sarcasmo: porque el autor a las claras se esconde detrás de la postura de Havelaar ${ }^{10}$.

\section{Contra la doble moral, postura colectiva}

La misma actitud de doble moral, llevada de lo individual a la política de Estado, se perfila detrás de otro personaje: Blatherer, en la

$\overrightarrow{10 .}$ En el segundo aporte sobre esta novela se visualizará como creación literaria y como, en el original en neerlandés, los apellidos de los persona jes, llevan una carga simbólica: "Droogstoppel" se traduce literalmente como "milpa seca, inútil", mientras "Havelaar" significa "el que siembre sabiamente". 
versión inglesa ${ }^{11}$. En español podríamos figurarnos ese individuo, nuevamente con toda ironía, como "Hablador". En efecto, detrás de su ropaje religioso y su palabrería (como sugiere su nombre), encontramos un perfecto ideólogo al servicio de la explotación. En un sermón, para probar el amor de Dios a partir de un pasaje de la Biblia (I Sam. $\mathrm{XV}: 33$ ), se deja decir: "Tal es, mis amados, la gloriosa misión de Israel! - y se refería a la exterminación de los habitantes de Canaány ¡también tal es la misión de Holanda! (...) Nuestro pueblo de Holanda ha sido escogido para salvar..."(139-140). También inventa Blatherer que "Dios ordena todo de tal modo que el ser bien pensante lleva a la riqueza" (esa y otras citas, 251). Desde luego, nuevamente, como contrapunto a este personaje tenemos al narrador.

La postura individual, tipo Droogstoppel, y la colectiva-estatal, tipo Blatherer, se juntan, reforzándose mutuamente. Las "medias tintas" y la doble moral se dan la mano. Otra interpretación religiosa y política al mismo tiempo del "burgués bien pensante" de Droogstoppel sería: "Si los indonesios son pobres es porque son incultos. Mientras los holandeses tengan que ver con Indonesia, más riqueza habrá aquí (en Holanda) y más pobreza allá. ¡Dios lo ha querido así!” Es, en términos estrictamente calvinistas, la creencia en la legitimidad de la simonía: por su trabajo uno podría comprar el cielo. Poco más abajo, vuelve a la carga el "religioso bien pensante" Blatherer: "Dios ordena todo de tal modo que lo correcto lleve a lo rico. (...) ¿No constituye eso claramente el dedo de Dios que el pobre trabaje para mantener al justo?" (citas varias, 251). Pero también hay otro dúo que se refuerza: el del narrador principal y el autor, para quienes tiene más peso otra afirmación bíblica: "por sus actos los conoceréis".

1. Lo mismo que con Droogstoppel (ver nota anterior), conviene recordar la carga simbólica, a la manera de Dickens, de los personajes. "Blatherer", en inglés está por "Wavelaar", en neerlandés, lo propongo también como "hablantín" o "parlanchín". 
Denuncia anticipada de la religión como "aparato ideológico del Estado"

No es que las dos posturas examinadas recientemente no existieran en tiempos del gran Bartolomé y, de seguro, en su obra deben constar de parte de él denuncias en contra de esas prácticas. Pero lo nuevo, en el caso de Multatuli es que él las evidencia de manera tan explícita, en su perversidad individual y más cuando son usadas en alianza táctica: todo su monumental alegato contra el sistema colonial holandés, flagrante opresor en Indonesia, se basa en ello. Lo nuevo también reside en que esas conductas se encuentran legitimadas mucho más fuertemente a través del protestantismo, mientras que, en tiempos de Las Casas, apenas empezaban a ser realidad.

Multatuli no duda en denunciar entonces la religión, no en sí, sino en tanto usada como práctica legitimadora de flagrantes atropellos. Tampoco ha de entenderse que no existiera esa práctica en tiempos de don Bartolomé: pareciera bastante sensato deducir que el abuso de la religión se presentó asociado al mismo surgimiento de la especie humana. En la misma época del "Protector de los indios" se presentaron casos históricos dramáticos, relevantes para América Central, como lo son los de Cortés, de Alvarado y de Pedrarias, entre otros, utilizando en forma maquiavélica la religión a su favor ${ }^{12}$. Lo cierto es que la conciencia respecto de este mecanismo ha ido aumentando, y el escritor contribuyó a ello. Modernamente, y a partir de Althusser ${ }^{13}$ se utiliza la terminología de "Aparato Ideológico del Estado" (abreviado: AIE) para este tipo de manipulación, entre otros de la religión, a favor del Estado.

12. Ver mi texto "Réquiem por Castilla del Oro: cobija política nicaragüense con ribetes trasatlánticos y flecos hasta nuestros días". Texto of recido a la revista Letras, de la Universidad Nacional, Heredia. Es un trabajo, esencialmente sobre interferencias belgas en la novela de Julio Valle, pero donde el autor desmonta con agilidad ese mecanismo del abuso de la religión por los primeros conquistadores, en Centroamérica.

13. El aporte del pensamiento marxista en el asunto es innegable. Ahora bien, si aquella frase de "la religión es el opio del pueblo" ya apuntaba a lo mismo, y es del Manifiesto Comunista (1847), no veo indicio alguno de influencia de Marx en Multatuli. 
todavía. Pero a partir de lo poco que uno, por desgracia, conoce de la literatura oriental y específicamente de este autor, en el Discurso de Lebak observa un paralelo soplo lírico, una similar relación entre hombre y naturaleza y sobre todo, un afán que también se encontrará a cada rato en Tagore, de lograr al menos interferencias entre la visión de mundo oriental y la occidental. Respecto de la influencia cristiana, ésta se perfila desde la misma educación familiar y escolar de Multatuli y conlleva una aplicación directa e integral de lo aprendido a lo vivido.

Según Multatuli se impone la línea del amor, amor por el prójimo, lo cual supone el aprendizaje "hacia el otro". Es decir, a pesar del choque intercultural, es posible buscar el trato justo, el humanismo más allá de la cáscara. Veamos una parte, al inicio del famoso discurso:

...siento alegría en mi corazón al verlos a todos reunidos aquí, para escuchar las palabras de mi boca. No ignoro que entre ustedes los hay con más sabiduría y más bondad en el corazón. Confío que a mi conocimiento le podré añadir del de ustedes, porque mi equipaje al respecto no es tan grande como hubiera deseado. Y pese a que adoro la bondad, se me pone en evidencia que persisten errores en mí que le dan sombra a la bondad de mi corazón y le condicionan su crecimiento. Todos ustedes saben que el árbol grande opaca al pequeño y lo mata. Por eso miraré hacia aquellos entre ustedes que sobresalen en virtud, en procura de crecer yo mismo en bondad (115).

¡Qué mensaje más actual de voluntad de acercamiento, de búsqueda de comprensión mutua, en vez de la prepotencia económica, ideológica y hasta militarista que prevalece de Occidente sobre Oriente! Nada de prepotencia jerárquica, racional-sabihonda. Con las múltiples invocaciones al corazón prevalece una clara función apelativa del mensaje. 


\section{Max Havelaar y el comercio justo, ahora}

Invito a una experiencia histórica y literaria: a como lo hice, leer o volver a leer a Multatuli, pero desde la perspectiva lascasiana; inversamente, leer el estudio de Durán, sobre don Bartolomé, pero a la luz de Multatuli. La confrontación es enriquecedora, en ambos sentidos. Ahora bien, "desde el límite/ de la agonía fundas la esperanza", le homenajea Pablo Neruda al español, en su Canto General: resulta una frase también perfectamente aplicable a Multatuli. Retomo mi calificativo de protohombres: Las Casas denunció por escrito terribles atropellos, una generación antes de pensadores humanistas de la altura de Montaigne, por ejemplo; a raíz de lo mismo, Multatuli hizo lo suyo, una generación antes de literatos de la talla de Zola, por ejemplo. ¡Grandes precursores fueron, los dos, en batallas que todavía no han terminado!

Pero hagamos ahora otro salto en el tiempo: redondeando, quinientos años han pasado desde la quijotada del dominico, ciento cincuenta desde la otra del apóstol laico: la lucha por el humanismo sigue. Nos hallamos confrontados con otro momento histórico importante: en gran parte resultado de un mundo unipolar, en lo político, económico y militar, en parte producto de una verdadera revolución tecnológica, sobre todo con lo digital. En todas partes, tanto en el Primer Mundo, como en el Tercero, vivimos embates y cambios vertiginosos. Ello ha traído consigo hasta una nueva vivencia de imperios y de empuje imperial: ahora son más disfrazados, sin consideración de fronteras estatales, de carácter transnacional. El Tratado de Libre Comercio (TLC) constituye un empeño jurídico norteamericano por estrechar vínculos mercantiles con América Latina. Ello parece loable, lo mismo que otros grupos geopolíticos, como la Unión Europea, por ejemplo, hacen lo suyo. Ahora bien, esa creciente formación de bloques en el ámbito global no puede implicar, pese al nombre eufemístico, un comercio mal tratado, nada libre, o para formularlo como el colega: "unos le llaman libertad al libre 
mercado cuando es ventajoso solo para ellos"16. A la inversa, de este lado de la barrera, ingenuamente muchos piensan que los otros se van a adaptar a los latinos o latinoamericanos porque son tan especiales y simpáticos en sí... Son dos posiciones extremas, por un lado, los acólitos incondicionales del neoimperialismo; por otro lado, la posición cerrada, "nacionalista" o "sindicalista" como muchos la viven, no por maduración, sino por miedo a un cambio inevitable.

Por eso, conviene también leer Max Havelaar en clave de TLC 0 , si se quiere, hacer el ejercicio inverso, leer el TLC en clave de Max Havelaar. Multatuli, el que allá lejos en el tiempo y en el espacio sufrió mucho por cuestiones de comercio injusto, parece guiñarnos de un ojo cuando escribe: "que mi relato sea de utilidad para el lector más tarde, cuando nubes oscuras se amontonen sobre su cabeza" (222). De verdad que en su novela se encuentran frases que todavía golpean la mente, en el contexto al que nos enfrentamos, comoaquellode que hay que evitar la "filantropía... sobre el papel" (216 y 228). Lo mismo que "es más fácil robar un país que robar un molino" (194): como que recientemente alguien robó un país, primero con la excusa de unas armas de destrucción masiva, cosa que después su mismo Secretario de Estado para Asuntos Externos desmintió. Con razón adquiere visos de ciudadanía la expresión anglosajona de "doble track": cubre grandemente lo que aquí se ha analizado desde esas dos formas de insinceridad examinadas, las "medias tintas" y la "doble moral" que se denunciaron en la novela.

Han pasado muchas décadas desde cuando Multatuli, en Bruselas, lanzó su grito. Con desesperación constataba, en lo inmediato: "me dejaron solo, pobre y sin poder en mi deseo de hacer justicia y llevar humanismo" (313). Pero al comprobar la extraordinaria resonancia local y universal de su novela, al ver que el nombre Max Havelaar, ahora no sólo implica una referencia literaria sino también

16. Victor Alba de la Vega, Suplemento Áncora, de La Nación, 25 de abril de 2004. 
toda una estructura defensiva de precios justos para productores ${ }^{17}$, su esfuerzo no habrá sido en vano. Multatuli, aunque sea inconscientemente, ha ahondado en el mismo surco de Las Casas y ya otros tomaron (¿tomamos?) el relevo. En Max Havelaar, la frase que más me ha golpeado, desde la perspectiva que nos ocupa, el "proyecto de construir una Nación Humana y Universal", es la que puse en el epígrafe. El drama de los holandeses contemporáneos a Multatuli es que solo entendieron de explotación, su propia ventaja en lo inmediato, como rapaces. La filosofía que propugna Multatuli era y es de interés mutuo, y a mediano y largo plazo.

17. El éxito alcanzado por las Fundaciones Max Havelaar legal y financieramente independientes en Suiza, Bélgica y Holanda ha conducido al establecimiento de organizaciones de comercio justo en otros países del mundo (específicamente Max Havelaar en Francia, Dinamarca y Noruega, TransFair en Alemania, Italia, Austria, Estados Unidos, Luxemburgo, Canadá y Japón o Fairtrade Foundation en Gran Bretaña, Irlanda, Rättvisemärkt en Finlandia y Suecia). El propósito de este tipo de fundaciones es promover el comercio con productores en desventaja y trabajadores de regiones en desarrollo, asegurándoles un ingreso que les permita vivir y al mismo tiempo ayuden con respecto al ambiente y se les asesora sobre asuntos relacionados con el desarrollo. La Fundación Max Havelaar otorga su etiqueta como una marca de distinción a productos de comercio-justo. Para Centroamérica vale la pena destacar la Fairtrade Labelling Organizations International (FLO). 